Progress in Chemical and Biochemical Research

Journal homepage: www.pcbiochemres.com

\title{
Sorptive chelation of metals by inorganic functionalized organic WOx-EDA nanowires: adsorbent characterization and isotherm studies
}

\author{
Adams Udoji Itodo $\mathbb{D}^{\mathrm{a}, *}$,Ohemu Michael Itodo ${ }^{\mathrm{a}}$, Esther Iornumbe a, Michael Omotola Fayomi ${ }^{\mathrm{a}}$ \\ aDepartment of Chemistry, Federal University of Agriculture, PMB 2373 Makurdi, Nigeria
}

\section{H I G H L I G H T S}

- The quest is to obtain sorption synergy from derived adsorbent with integrated organic and inorganic functionalities.

- A simple solvo-thermal method is reported for synthesis of the characterized WOx-EDA nanowires (adsorbent).

- Results of adsorption properties indicated the potential drug candidature of WOx-EDA for the chelating treatment of $\mathrm{Pb}$ and $\mathrm{Cu}$ poisoning.

\section{A R T I C L E INF O}

\section{Article history:}

Received: 16 December 2018

Accepted: 13 January 2019

Available online: 18 January 2019

Manuscript ID: PCBR-1810-1009

\begin{tabular}{l}
\hline Keywords \\
Adsorption \\
Tungsten oxide-ethylenediamine \\
Metals \\
Copper \\
Lead \\
Isotherm \\
Nanotubes \\
Nanobelts
\end{tabular}

G R A P H I C A A B S T R A C T

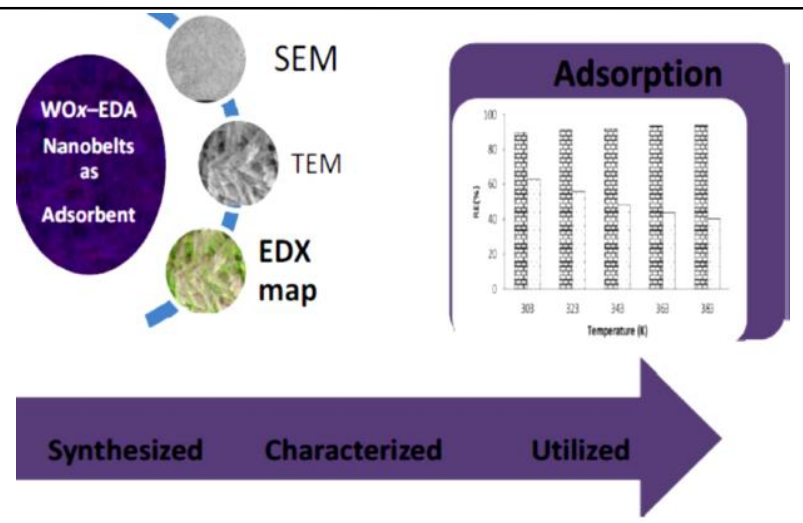

\begin{abstract}
A B S T R A C T
Synthesis and Characterization of Inorganic-organic hybrid Tungsten oxideethylenediamine (WO $x$-EDA) nanowires have been carried out by a simple, low-cost and high-yield solvo-thermal method. The WOx-EDA nanowire adsorbent was characterized using, Fourier-transform infrared (FT-IR) spectroscopy, scanning electron microscopy (SEM), transmission electron microscopy (TEM) and Energy Dispersive X-ray spectroscopy (EDX). Adsorptive potential of the hybrid for aqueous phase copper and lead removal was investigated using batch adsorption process. The equilibrium data were subjected to various isotherm models. The Langmuir model shows high applicability ( $\mathrm{R}^{2}$ of 0.921 ) for lead uptake. Other $\mathrm{R}^{2}$ values include 0.882 (for Freundlich model), 0.947 (Temkin) and 0.972 (Dubinin- Radushkevich). Similarly, adsorption experimental runs for $\mathrm{Cu}$ uptake gave correlation coefficient $\left(\mathrm{R}^{2}\right)$ values of $0.972,0.584,0.906$ and 0.899 for the Langmuir, Freundlich, Temkin and Dubinin- Radushkevich isotherm models respectively. Overall, Tungsten oxide ethylenediamine adsorbent proved its potential candidature for the chelating treatment of $\mathrm{Pb}$ and $\mathrm{Cu}$ poisoning as well as purifications of water containing such metals.
\end{abstract}

\section{Introduction}

Metal poisoning is the toxic effect of heavy metals on life. Toxic metals are significant environmental pollutants whose toxicity poses health challenges of increasing significance. In March, 2010, it was reported in Zamfara state, Northern Nigeria, the death of an estimated 400 children linked to lead poisoning [1]. Toxic metals can be removed from environment through complexation, ion exchange, chelation and adsorption or by combination of the mechanism. In adsorption, adsorbate (a gas or liquid solute) accumulates on the surface on an adsorbent (liquid or solid).

Adsorption isotherms are important to clarify the interaction of adsorbate to adsorbents, and optimize their usage condition. Correlation of equilibrium data using a 
theoretical or empirical equation is useful for adsorption data interpretation and prediction.

In the context of this study, nanocomposites described as either nanowires, nanobelts, nanocrystals or nanoparticles (depending on their microstructural morphology, surface morphology and functionality) are nanostructures, with the diameter of the order of a nanometer $\left(10^{-9}\right.$ meters). The adsorbability of metal ions to nanomaterials is very strong due of their large surface area and surface multi-unsaturated atoms and because the cost of metal oxides such as $\mathrm{Al}_{2} \mathrm{O}_{3}, \mathrm{CeO}_{2}, \mathrm{TiO}_{2}, \mathrm{MgO}, \mathrm{Fe}_{2} \mathrm{O}_{3}$, and $\mathrm{Fe}_{3} \mathrm{O}_{4}$ was vile and their preparation was easy, the study on the adsorption of metal ions on nanometer metal oxides was widely attended to [2]

Chelation therapy is a preferred medical remedy for detoxifying effects of metals in human system. Agents of Chelation are capable of binding metal ions, forming complex structures that are easily excreted from the body. Reports shows that introduction of the medical use of EDTA as a lead chelating agent was after World War II due to mass lead poisoning observed in large number of navy personnel, as a result of their routine task of repainting the hulls of ships [3].

Several mathematical models are applicable in describing experimental data of adsorption isotherms. Equations 1,2,3 and 4 represents the various forms of the Langmuir equations $[4,5]$.

$$
\begin{aligned}
& \frac{C_{e}}{q_{e}}=\frac{1}{b Q_{o}}+\frac{C_{e}}{Q_{o}} \\
& \frac{C_{e}}{q_{e}}=\frac{1}{Q_{o}}+\frac{C_{e}}{b Q_{o} C_{e}} \\
& q_{e}=Q_{o}-\frac{q_{e}}{b C_{e}} \\
& \frac{q_{e}}{C_{e}}=b Q_{o}-b q_{e}
\end{aligned}
$$

Their respective plot areas are $\frac{C_{e}}{q_{e}}$ vs $C_{e}, \frac{1}{q_{e}}$ vs $\frac{1}{C_{e}}, q_{e}$ vs $\frac{q_{e}}{C_{e}}$ and $\frac{q_{e}}{C_{e}}$ vs $q_{e}$. Langmuir empirical model assumes monolayer adsorption at a fixed number of definite localized sites, that are identical and equivalent in its derivation; $\mathrm{K}_{\mathrm{L}}(\mathrm{L} / \mathrm{mg})$ and $\mathrm{q}_{\mathrm{m}}$ are Langmuir isotherm constants

Freundlich model (Equation 5) proposes heterogeneous energetic distribution of active sites; $\mathrm{Q}_{\mathrm{e}}(\mathrm{mg} / \mathrm{g})=$ adsorbate adsorbed per unit mass of the adsorbent and $\mathrm{C}_{\mathrm{e}}(\mathrm{mg} / \mathrm{L})=$ equilibrium concentration. Intercept, $\mathrm{K}_{\mathrm{f}}=$ constant related to the adsorption capacity (Freundlian constant) and slope, $\mathrm{n}_{\mathrm{f}}$ is an empirical parameter related to be adsorption intensity which varies with the heterogeneity of the materials. A plot of $\ln \mathrm{Q}_{\mathrm{e}}$ vs $\ln C_{e}$ results to good linearity representing fitness of process to the model [7].

$$
\ln Q_{e}=\frac{1}{n} \ln \mathrm{C}_{e}+l_{n} K_{f}
$$

Temkin model (Equation 6) states that the heat of adsorption of all the molecules will increase linearly with coverage; $b_{\mathrm{T}}$ is Tempkin constant, deduced from the plot of $q_{e}$ vs $\ln C_{e}$. R is the universal gas constant $(8.314 \mathrm{~J} / \mathrm{molK})$, $\mathrm{T}=$ is absolute temperature (K) [7].

$$
q_{e}=\frac{R T}{b_{T}} \ln a_{T}+\frac{R T}{b_{T}} \ln C_{e}
$$

Dubinin- Radushkevich isotherm gave B as a constant related to the mean free energy of adsorption per mole of the adsorbate $\left(\mathrm{mol}^{2} / \mathrm{J}^{2}\right), \mathrm{q}_{\mathrm{D}}=$ theoretical saturation capacity $\left(\mathrm{mg} \mathrm{g}^{-1}\right)$, and $\varepsilon=$ polanyi potential, $\mathrm{E}$ the mean sorption energy that predict whether an adsorption is physi-sorption or chemi-sorption.

$$
\ln q_{e}=\ln q_{D}-B_{\varepsilon^{2}}
$$

For a good model applicability with high correlation coefficient, a plot of $\ln \left(q_{e}\right) v s \varepsilon^{2}$ will be linear. The polanyi potential and mean sorption energy are given as equations 8 and 9 respectively [8].

\subsection{Inorganic functionalized organic hybrid WOx-EDA} nanowires

Metal oxide adsorbents $\left(\mathrm{Al}_{2} \mathrm{O}_{3}, \mathrm{CeO}_{2}, \mathrm{TiO}_{2}, \mathrm{MgO}, \mathrm{Fe}_{2} \mathrm{O}_{3}\right.$, and $\left.\mathrm{Fe}_{3} \mathrm{O}_{4}\right)$ and binary oxides $\left(\mathrm{Al}_{2} \mathrm{O}_{3} / \mathrm{Fe}_{2} \mathrm{O}_{3}\right.$ and $\left.\mathrm{TiO}_{2} / \mathrm{ZrO}_{2}\right)$ have been reported to exhibit low adsorption properties owing to relatively weak affinity for heavy metal [9]. On the contrary, organic functional groups (amino, carboxylic, thiol) and sulfur groups exhibit stronger complexation or chelating ability towards heavy metal ions, and have been used to functionalize the inorganic adsorbents to improve their adsorption performance [10]. Examples are derived nanostructured inorganic-organic materials such as $\mathrm{WO}_{2}$ (deferoxamine) $\quad\left(\mathrm{C}_{7} \mathrm{H}_{6} \mathrm{O}\right)$ nanowires $\mathrm{ZnS} /$ amine nanowire, GeOx/ethylenediamine nanowires, $\mathrm{Zn}_{2} \mathrm{GeO}$ /ethylenediamine nanoribbons, ZnSe(diethylenetriamine) nanobelts, and CoSez/amine nanobelts [11]. Expectations are the combine functional organic components and inorganic building to integrate the superior rigidity and thermal stability of the inorganic framework with new flexibility, structural diversity, and functionalities, thereby striking a synergy [12].

This present study did not focus on the usual electronic and optical properties of hybrid composite, but on the synthesis, characterization and the adsorptive properties of Tungsten oxide ethylenediammine adsorbent (an inorganic functionalized organic hybrid materials) for the 
aqueous phase detoxification of simulated system containing $\mathrm{Pb}$ and $\mathrm{Cu}$ ions. The adsorptive properties of derived adsorbent were based on isotherm studies. Sorbent characterization was achieved using FT-IR analysis; Transmission electron microscope, Energy dispersive X-ray spectroscopy (EDX), Scanning electron microscope, ultra violet-visible analysis and toxic metals level were quantified using AAS.

\section{Experimental}

\subsection{Reagents}

Analytical grade ammonium tungstate, with molecular formula $\left(\mathrm{NH}_{4}\right)_{10} \mathrm{H}_{2} \mathrm{~W}_{12} \mathrm{O}_{42}$, ethylenediamine were procured and used as received. Others include distilled water, lead salt $\left(\mathrm{Pb}\left(\mathrm{NO}_{3}\right)_{2}\right)$, copper salt $\left(\mathrm{CuSO}_{4} .5 \mathrm{H}_{2} \mathrm{O}\right)$ and ethanol.

\subsection{Synthesis of WOx-EDA hybrid nanowires}

A facile solvothermal procedure was followed for the synthesis of WOx-EDA hybrid nanowires. In a typical protocol, mixed $15.1 \mathrm{~g}$ ammonium tungstate $\left(\left(\mathrm{NH}_{4}\right) 10 \mathrm{H}_{2} \mathrm{~W}_{12} \mathrm{O}_{42} \cdot 4 \mathrm{H}_{2} \mathrm{O}\right.$ and $60 \mathrm{~mL}$ ethylenediamine were vigorously stirred using magnetic stirrer for $10 \mathrm{~min}$ and transferred to a Teflon-lined stainless steel autoclave with an inner volume of $100 \mathrm{~mL}$. Mixture was heated to $200{ }^{\circ} \mathrm{C}$ for $24 \mathrm{~h}$, followed by natural cooling to room temperature. The product was collected by centrifugation and washed thoroughly with deionized water until a near neutral $\mathrm{pH}$ range was attained. Washing was carried out with small several portions of ethanol to remove possible residues followed by vacuum oven drying at $60{ }^{\circ} \mathrm{C}$ overnight [13].

\subsection{Preparation of saturated solutions.}

The saturated solutions of 2-amino-4,5dimethylthiophene-3-carboxamide (ADTC) in mixed EtOH$\mathrm{H}_{2} \mathrm{O}$ solvents were prepared by dissolving solid material (ADTC) in closed test tubes containing different mole fractions of ethanol-water solvents. The tubes were put in water thermostat of type assistant for a period of one day at temperatures 293.15, 298.15, 303.15, 308.15, and $313.15 \mathrm{~K}$ till equilibrium reached.

The solubility of (ADTC) was measured by taking $1 \mathrm{ml}$ of each saturated solution putting in a small weighed beaker $(10 \mathrm{~mL})$ and evaporate under I.R. lamp till dryness and then weight [15-17].

\subsection{Characterization of Derived Adsorbent}

Derived adsorbent was characterized using Fouriertransform infra-red spectrometer (FT-IR; Agilent technology) to investigate active functional groups, scanning electron microscopy (SEM) for surface morphology, Energy Dispersive X-ray spectrometer (Carl Zeiss) for elemental analysis. Transmission electron microscopy (TEM) was used to examine the microstructural and compositional characteristics of the nanocomposites such as grain size, crystallinity and chemical variations at a resolution down to the nanometer scale. Divalent metal ion quantification was achieved by Atomic Absorption Spectrometer (AAS). Metal ion stock and standard solutions were prepared using standard procedures.

\subsection{Batch Adsorption Experiments}

The experiment based on slight modification of existing method [14] was carried out by shaking using a mechanical shaker for $120 \mathrm{~min}$ at room temperature. The adsorbent (0.03 g) was missed with $50 \mathrm{~mL}$ of metal ion solution (whose $\mathrm{pH}$ was adjusted to $\mathrm{pH} 4$ with $\mathrm{NaOH}$ or $\mathrm{HCl}$ solution using a pH meter). $10 \mathrm{~mL}$ of the filtrate was collected for estimation of equilibrium phase (residual or un adsorbed) concentrations of heavy metal ion by means of atomic absorption spectroscopy (AAS).

\subsection{Investigating the Effect of Parametric Factors}

For effect of initial metal concentration, each of the $1000 \mathrm{mg} / \mathrm{L}$ stocks of $\mathrm{Cu}^{2+}$ and $\mathrm{Pb}^{2+}$ prepared from their respective salts $\left(\mathrm{CuSO}_{4} .5 \mathrm{H}_{2} \mathrm{O}\right.$ and $\left.\mathrm{Pb}\left(\mathrm{NO}_{3}\right)_{2}\right)$ were used to prepare series of working standards $(10,20,30,40$ and 50 $\mathrm{mg} / \mathrm{L}$ ). $0.03 \mathrm{~g}$ adsorbent was added to $50 \mathrm{mLCu}^{2+}$ and $\mathrm{Pb}^{2+}$ aqueous solutions. The mixture was shaken for 120 min and the filtrate gotten using centrifuge and filtration, and then the metal concentration was analysed using AAS. Also investigated were effect of $\mathrm{pH}$ [15], dosage; 0.01, 0.03, $0.05,0.07$ and $0.09 \mathrm{~g}$ and dwell time; 60, 120, 180, 240 and $300 \mathrm{~min}$

\subsection{Isotherm Studies}

Data generated from adsorption experimental runs for the effect of initial sorbate concentration was subjected to different isotherm models namely, Langmuir isotherm model, Freundlich adsorption isotherms, Temkin Isotherm model, and Dubinin-Radushkevic. Adsorption phenomenon (capacity and intensity) was explained from model of best fit.

\section{Result and Discussion}

\subsection{Physicochemical Properties of the Complex, WOx-} $E D A$

Results of physicochemical study are as follows; the high yield (89.4 \%) WOx-EDA solid shows yellowish brown after the synthesis, linking to one of the characteristics of 
transition metals which is the formation of colour complexes. The pungent smell of the complex is as the result of ammonia present in the ammonium tungstate which is the starting material in the reaction. The high melting point (308760C) is because a lot of energy is needed to break the strong metal oxide bond present in the complex [16].

\subsection{SEM characterization of WOX-EDA}

The surface morphology of the synthesized complex was studied by scanning electron microscopy. Fig. 1a, shows the scanning image of the synthesized WOx-EDA, Fig. 1b shows the image of the spent adsorbent $(\mathrm{Pb}$ on WOx-EDA), Fig. $1 \mathrm{c}$ is the image of Wox-EDA loaded with $\mathrm{Cu}$. The size and shape of the complex was examined using the scanning electron microscope. Fig. 1a displays a representative SEM image at 2500 magnification. It revealed that the product is composed of Nano belts with widths and lengths up to several tens of micrometers. Nano belt-like homogeneous morphologies are revealed as flat rocky structures with several holes and black spots showing adsorptive site at 1500 magnification. Fig. $1 \mathrm{~b}$ and 1c shows a smoother surface in comparison to the ones observed in Fig. 1a, this could be as a result of the adsorbates $(\mathrm{Pb}$ and $\mathrm{Cu})$ filling up the adsorptive sites. Similar results were observed [17].

\subsection{TEM characterization of WOx-EDA}

Furtherance on the microstructural form of WOx- EDA is confirmation by the projected mass-density contrast observed by transmission electron microscopy. Images of the product were obtained on a transmission electron microscope (TEM) and high resolution TEM (Carl Zeiss). Fig. 2 shows the TEM image of WOx-EDA.

(a)
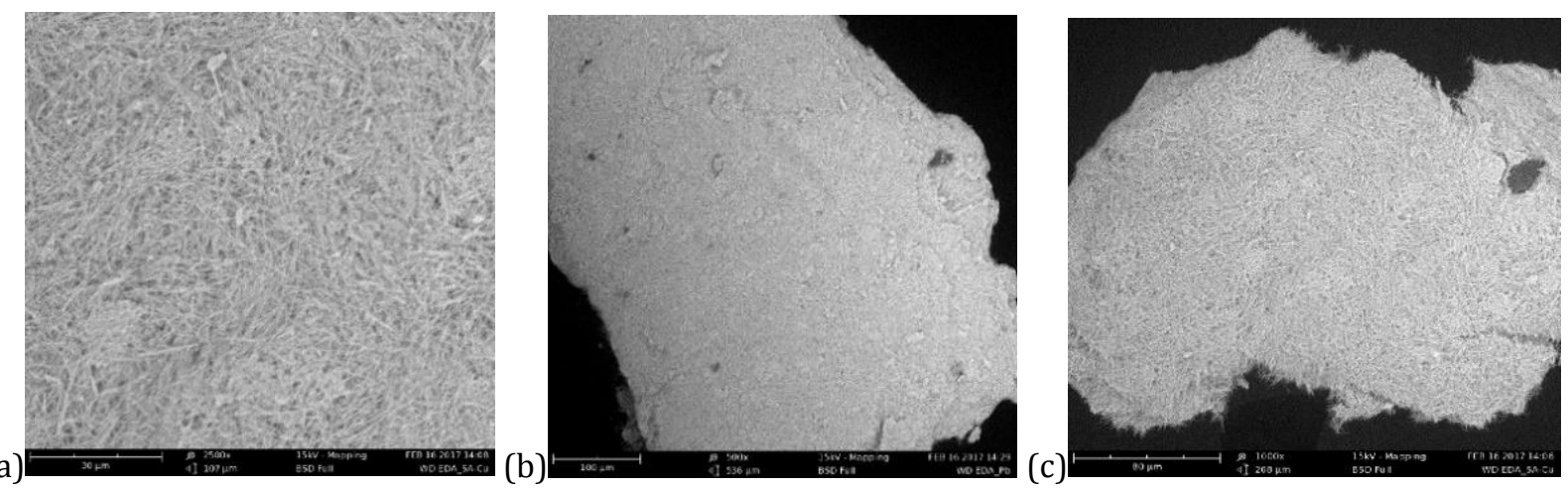

Fig. 1. SEM Micrograph of (a) WOx-EDA(b) Pb-WOx-EDA and (c) Cu-WOx-EDA at 2500X Magnification

TEM image in Fig. 2 indicates that the WOx-EDA nanowires are straight and uniform nanobelts. This, as opined in a study [17] indicated that their structures have thickness or diameter constrained to tens of nanometers or less and an unconstrained length and also they are composed of repeating molecular units.

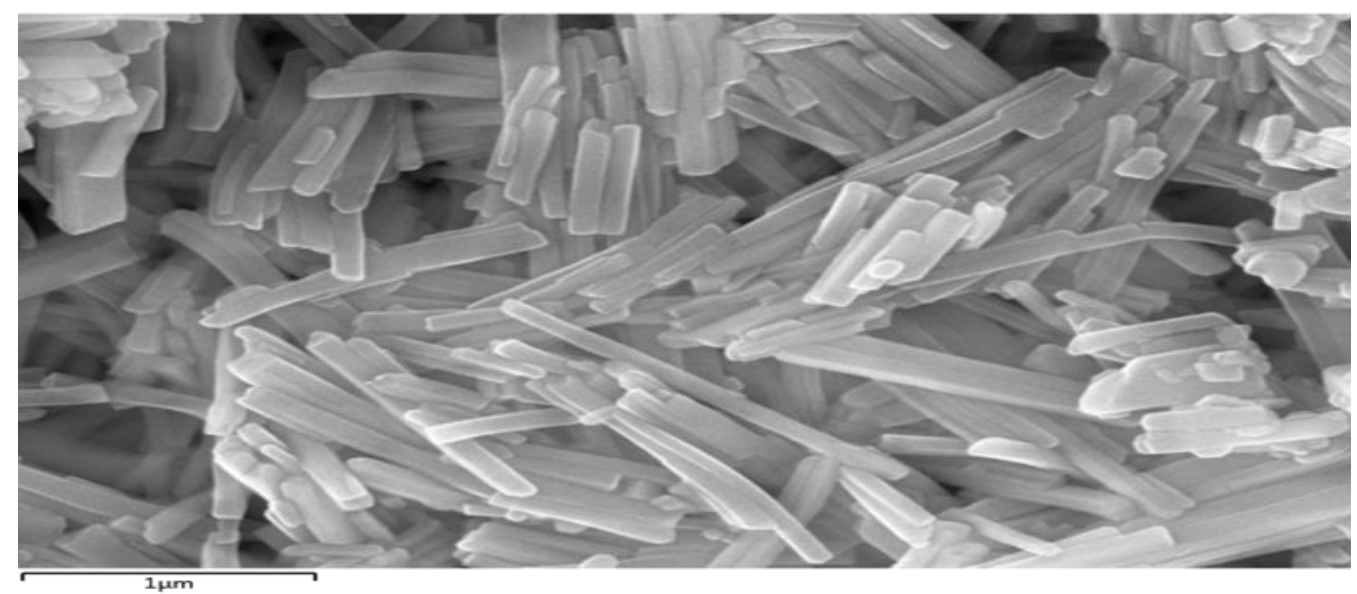

Fig. 2. TEM image of WOx-EDA Nanotubes 


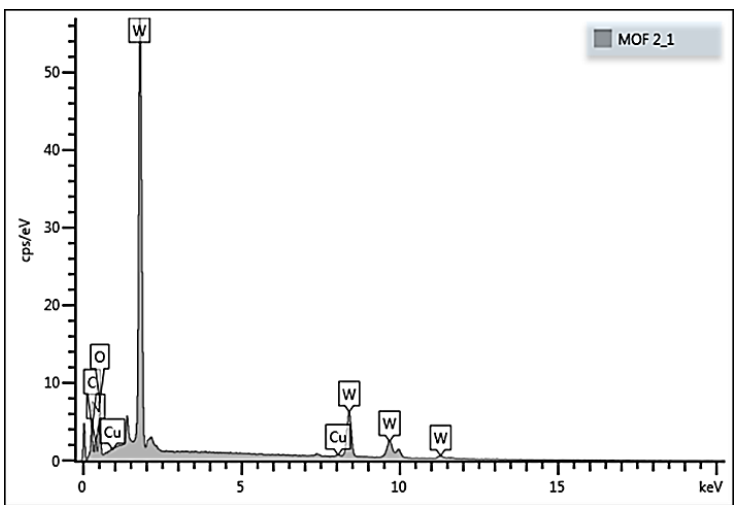

Fig. 3. EDX Spectrum taken from a Single Hybrid WOx-EDA Nanobelt

\subsection{Elemental analysis using Energy Dispersive X-ray} Spectroscopy (EDX)

The synthesized complex was subjected to EDX analysis to determine the elemental compositions of the synthesized inorganic. Fig. 3 shows the EDX spectrum taken from a single hybrid WOx-EDA nanobelts, indicating the presence of carbon, nitrogen, oxygen and tungsten. Fig. 3 also confirms the presence of $\mathrm{C}, \mathrm{N}, \mathrm{O}$, and $\mathrm{W}$. This is in conformity documented report [17]. The $\mathrm{C}, \mathrm{N}, \mathrm{O}$ shows the presence of the organic ligand chelated with the metal $\mathrm{W}$.
3.5 Qualitative elemental analysis using ultra plus FSEM

The elemental composition of WOx-EDA was also confirmed using Ultra Plus Field Emission scanning microscope. The individual elements in the derived WOxEDA presented using color mapping indicates $\mathrm{O}$ (oxygen); W (tungsten); C (carbon); $\mathrm{N}$ (Nitrogen) and $\mathrm{Cu}$ (copper) as shown in Fig. 4 (a-e). Fig. $4 \mathrm{f}$ shows the color images of the distribution of elements present in WOx-EDA. By inspection, tungsten was more prominent in the complex. This could be as a result of the tungsten being the central atom.

3.6 Quantitative Elemental Analysis using Ultra plus FSEM The quantitative analysis based on weight percent was confirmed. Percentage compositions of the elements present in WOx-EDA was estimated with Ultra Plus Field Emission Scanning Electron Microscopic quantification based on weight percent as shows tungsten, having the highest weight percent of $57.52 \%$. This could be as a result of metals being very heavy and are more abundant than the rest elements present [17].

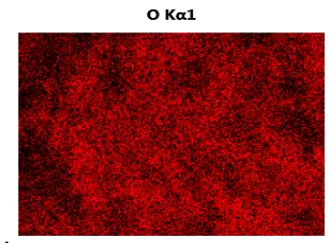

(a)

$\longdiv { \sqrt { 1 \mu m } }$

(d)

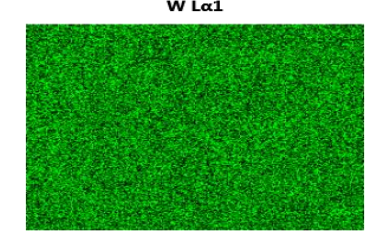

(b) $\longdiv { 1 , m }$

(c)

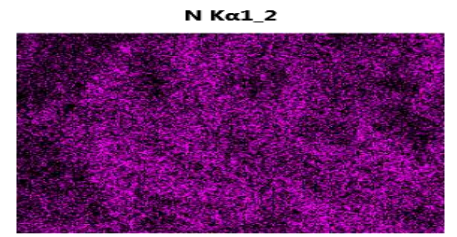

(c) $\longdiv { { } ^ { 1 \mu m } }$
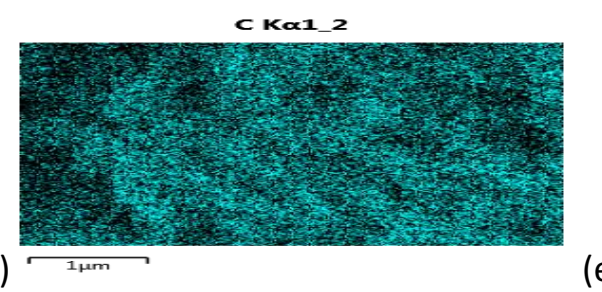

(e)

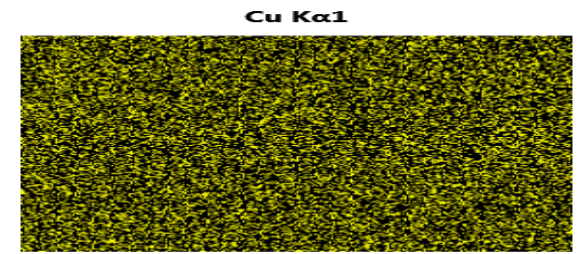

e) $\longdiv { 1 \mu m }$

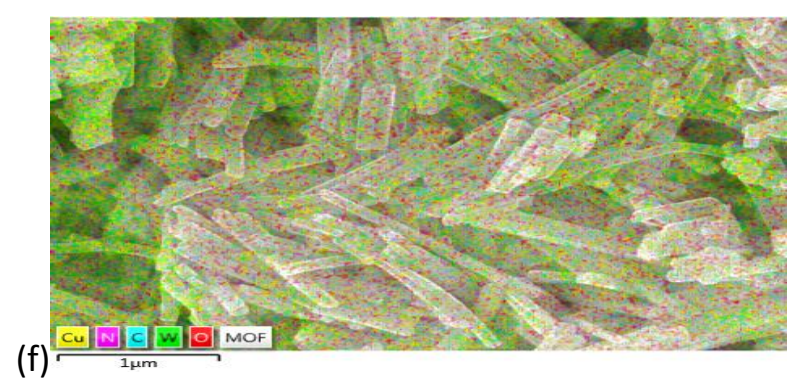

Fig. 4. SEM's EDS images of elements (a-e) and EDS map (f) showing distribution of elements in synthesized tungsten oxide ethylenediamine adsorbents. 
The percentage compositions of the elements present in WOx-EDA include carbon (15.22), nitrogen (10.77), oxygen (15.83), Cupper (0.66) and tungsten (57.52).

\subsection{Spectral Characteristic of Adsorbents}

The FT-IR spectrum for tungsten oxide ethylenediamine is shown as Fig. 5a Table 1 shows the FT-IR spectra characteristics of derived WOx-EDA and spent WOx-EDA. A band was observed at $3200 \mathrm{~cm}^{-1}$ which is attributed to $\mathrm{v}(\mathrm{O}$ H) stretching frequencies. Similar bands are observable in the spent adsorbents, but in higher frequencies, indicating that adsorption took place. The presence of the band at range around 1617.7 indicates the presence of $\mathrm{N}-\mathrm{H}$ bend; this is because of the ethylenediamine ligands. The bands in the spectra of the metal oxide are observed at $408.97 \mathrm{~cm}^{-1}$. The strong bands at 2961.25 and $2895.36 \mathrm{~cm}^{-1}$ are attributed to $\mathrm{v}(\mathrm{C}-\mathrm{H})$ stretching frequencies for co-ordination [13].

UV-visible Spectrum of WOx-EDA (Fig. 5b) revealed a sharp band-edge absorption peak at $274 \mathrm{~nm}$. The remarkably blue-shifted peak, as compared with peak for conventional tungsten oxide (350-450 nm), may be attributed to the strong quantum size effects in these novel hybrid nanobelts. Incorporation of ethylenediamine molecules, which act as barriers in the inorganic frameworks, could induce strong quantum confinement of both electrons and holes similarly to those in previous report [17] for ZnTe(EDA) and GeOx/EDA (a)

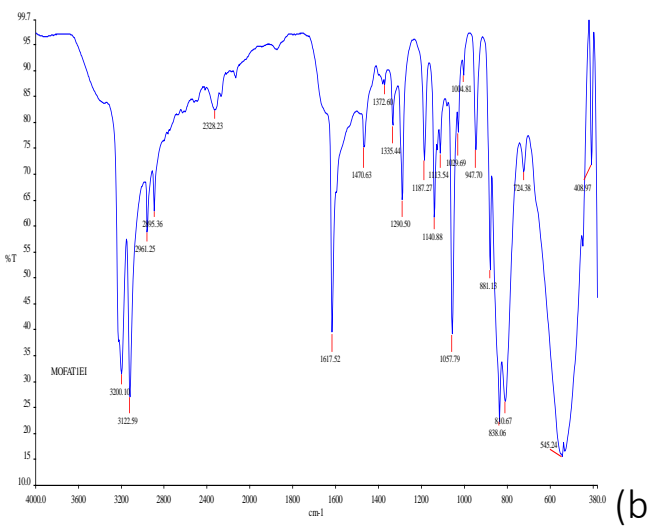

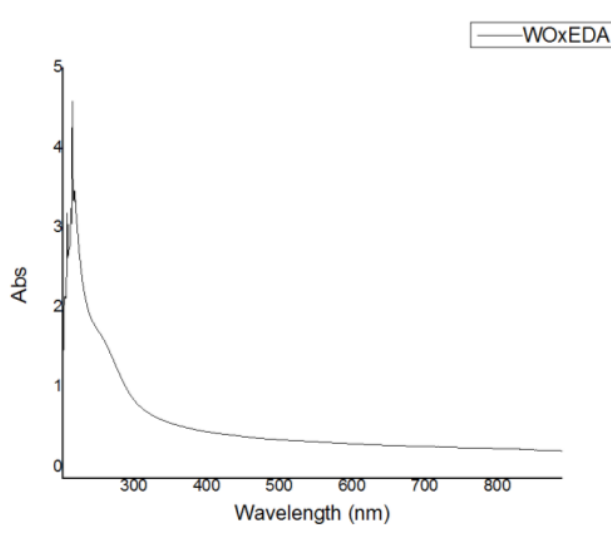

Fig. 5. (a) FT-IR and (b) UV-vis. Spectrum of Tungsten Oxide Ethylenediamine

Table 1. FT-IR Spectra Characteristics of Derived and Spent WOx-EDA

\begin{tabular}{|c|c|c|c|c|c|}
\hline \multirow[t]{2}{*}{ Group Frequency $\left(\mathrm{cm}^{-1}\right)$} & \multirow{2}{*}{$\begin{array}{l}\text { Characteristic } \\
\text { Functional Group }\end{array}$} & \multicolumn{3}{|c|}{ Observed Frequency $\left(\mathrm{cm}^{-1}\right)$} & \multirow{2}{*}{ Assignment } \\
\hline & & WOx-EDA & Pb-WOx-EDA & $\overrightarrow{\mathrm{Cu}}-$ WOx-EDA & \\
\hline $3300-2500$ & O-H Stretch & $3200,3122.5$ & $3205.3,3127$ & $3235-3131$ & Carboxylic acids \\
\hline $3000-2500$ & C-H Stretch & $2961.25,2895.36$ & $2963.2,2895$ & $2963.2-2895$ & Alkanes \\
\hline $1650-1580(\mathrm{~m})$ & $\mathrm{N}-\mathrm{H}$ bend & 1617.52 & 1617.7 & 1617.7 & Amines \\
\hline $1500-1400(\mathrm{~m})$ & C-C stretch (in ring) & 1470 & 1468 & 1468.8 & Aromatics \\
\hline $1250-1020(\mathrm{~m})$ & C-N stretch & 1140 & 1140.6 & 1140.6 & Aliphatic amines \\
\hline $\begin{array}{l}950-910(\mathrm{~m}) \\
900-675 \\
611-400\end{array}$ & $\begin{array}{l}\text { O-H bend } \\
\text { C-H } \\
\mathrm{M}-\mathrm{O}\end{array}$ & $\begin{array}{l}947 \\
881,838 \\
488.97\end{array}$ & $\begin{array}{l}946 \\
883,838.7\end{array}$ & $\begin{array}{l}946.6 \\
883,842.4\end{array}$ & $\begin{array}{l}\text { Carboxylic acids } \\
\text { Aromatics } \\
\text { Metal oxide }\end{array}$ \\
\hline
\end{tabular}

\subsection{Batch Adsorption Studies}

The effects of contact time, Adsorbent dosage, $\mathrm{pH}$, temperature and initial metal concentrations were studied. Fig. 6 shows effects of parametric factors on percentage removal of $\mathrm{Pb}$ and $\mathrm{Cu}$ onto WOx-EDA. Percentage removal increases as the adsorbent dosage increases both for $\mathrm{Pb}$ and $\mathrm{Cu}$ with the highest removal found to be at $0.09 \mathrm{~g}$ achieving the percentage removal of $93.5 \%$ and $99.2 \%$ respectively. This could be because of the introduction of more binding sites for adsorption.

\subsubsection{Effect of $p H$}

The result of $\mathrm{Pb}$ removal on WOx-EDA revealed that as $\mathrm{pH}$ increases from 2 to 4 , the percentage removal increase from $88.7 \%$ to $92 \% \%$ and adsorption capacity increases, 
this may be because of the decrease of removal of metal ion at low $\mathrm{pH}$ due to the higher concentration of $\mathrm{H}^{+}$ion in the solution which compete with $\mathrm{Pb}^{+}$for the adsorption site of adsorbents. As the $\mathrm{pH}$ value increases from 6 as in the case of $\mathrm{Cu}$ on WOx-EDA, the percentage removal and adsorption capacity also increases.

\subsubsection{Effect of contact time}

For the Pd-WOx-EDA experiment carried out with varying the contact time $(60,120,180,240$ and 300 mins), it was observed that the highest percentage removal was found at 300 mins achieving $95.7 \%$. This may be linked to active adsorption site being abundant. Similar report was documented by Oyedeji and Osinfade [18]. In the experiment that involved the adsorption of copper on WOxEDA, it was observed that as the contact time increases, the percentage removal of the copper ion also increases (Fig. 6). The fast adsorption at the initial stages is due to the availability of vacant active sites on the surface of the adsorbent [19].

\subsubsection{Effect of concentration}

It was observed that the percentage removal of the $\mathrm{Pb}$ increases as the concentration of the metal increases. Results suggested that, if the metal ion concentration in solution increases, the difference in concentration between bulk solution and surface also increases, intensifying the mass transfer processes. Accordingly, a higher quantity will be adsorbed, proving that WOx-EDA has a high adsorption capacity for metal ions. This corroborate other report [20]. The experiment investigated on the Adsorption of $\mathrm{Cu}$ by WOx-EDA also shows an increase in the percentage removal of copper as the initial metal concentration increases, and it peaked at $40 \mathrm{mg} / \mathrm{L}$. This decrease in copper removal percentage at $50 \mathrm{mg} / \mathrm{L}$ could be due to lack of sufficient active sites on WOx-EDA to adsorbed more metal ions available in the solution [21].
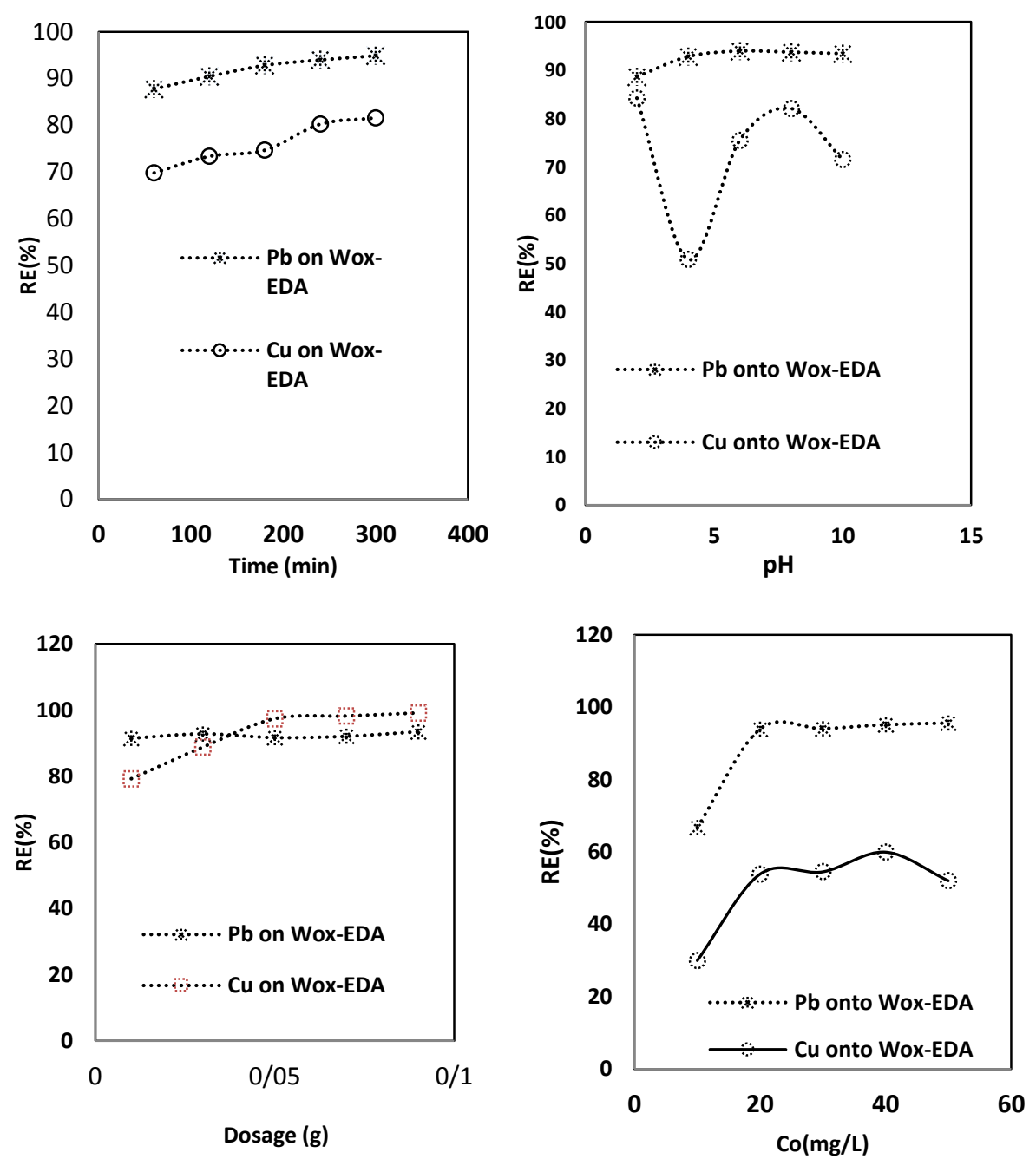

Fig. 6. Effects of Parametric Factors on Percentage Removal of $\mathrm{Pb}$ and $\mathrm{Cu}$ onto WOx-EDA 


\subsubsection{Effect of temperature}

To study the effect of temperature on the removal of $\mathrm{Pb}$, the experiment was carried out at temperature varying from $303 \mathrm{k}$ to $383 \mathrm{~K}$. The removal of $\mathrm{Pb}$ was found to be highest at 383K (93.6\%) and lowest at 303K (89.5\%) for WOx-EDA and Fig. 7 shows that, as the temperature increases the percentage removal of the metal also increases. This indicates that a high temperature favors the adsorption process. The improved adsorption capability with increasing temperature suggests that the adsorption is an endothermic one. This trend may be due to the tendency of $\mathrm{Pb}$ ions gaining more kinetic energy to diffuse from the bulk phase to the solid phase with an increase in solution temperature. Similar result was earlier observed [19].

In the experiment involving the removal of $\mathrm{Cu}$ by $\mathrm{WOx}-$ EDA. After varying the temperatures and keeping all the other parameters constant, it was observed that, as the temperature increases, the percentage removal of the $\mathrm{Cu}$ by WOx-EDA decreases (Fig. 7). The decrease could be as a result of increase in temperature that leads to increase in kinetic energy of the metal ions and therefore weakening the forces of attraction between the metal ions and the adsorbent. This suggests that the adsorption process is exothermic as similarly reported [18].

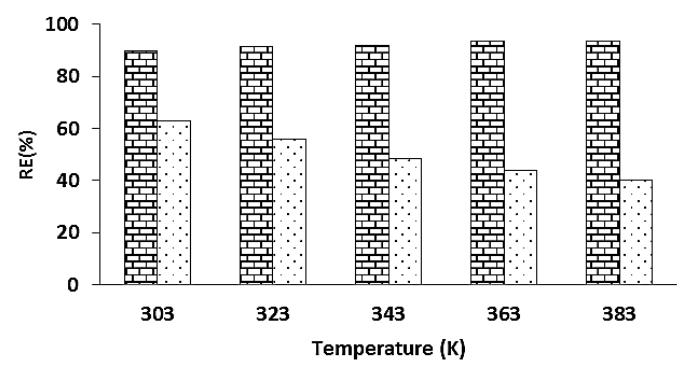

Fig. 7. Effects of Temperature on Removal Efficiency of $\mathrm{Pb}$ and $\mathrm{Cu}$ onto WOx-EDA

\subsection{Isotherm study}

The adsorption equilibrium data generated were fitted into isotherm models. The isotherm plots of $\mathrm{Pb}$ and $\mathrm{Cu}$ on WOx-EDA is shown in Fig. 8.

Table 2 represents the isotherm parameters showing the adsorption of $\mathrm{Pb}$ and $\mathrm{Cu}$ onto WOx-EDA. The adsorption isotherm indicates how the adsorption molecules distribute between the liquid-Phase when the adsorption process reaches an equilibrium state [22]. In general, an adsorption isotherm is an invaluable curve describing the phenomenon governing the retention (or release) or mobility of a substance from the aqueous porous media or aquatic environments to a solid-phase at a constant temperature and $\mathrm{pH}[22,23]$. Data generated were fitted into four isotherm models namely; Langmuir, Freundlich, Temkin, Dubinin-Radushkevich.

In Langmuir theory, it is assumed that adsorption takes place at specific homogeneous sites within the adsorbent. A linear plot obtained for Langmuir isotherm is shown in Fig. 8 , with values of $\mathrm{R}^{2}$ for $\mathrm{Pb}$ and $\mathrm{Cu}$ on WOx-EDA as 0.921 and
0.972 respectively. This shows a good applicability of the model, indicating monolayer adsorption.

The essential feature of the Langmuir isotherm can be expressed by means of dimensionless constant separation factor $\left(\mathrm{R}_{\mathrm{L}}\right)$. In this context; low $\mathrm{R}_{\mathrm{L}}$ values reflects favorable adsorption. Generally, $\mathrm{R}_{\mathrm{L}}$ value indicates the adsorption nature to be either unfavourable $\left(R_{L}>1\right)$, linear $\left(R_{L}=1\right)$, favourable $\left(0<R_{L}<1\right)$ or irreversible $\left(R_{L}=0\right)$

From Table 2, $\mathrm{R}_{\mathrm{L}}$ for both Pband $\mathrm{Cu}$ on WOx-EDA are less than 0 , indicating favourable adsorption. Similar to this finding is the report of Hameed and Foo [24].
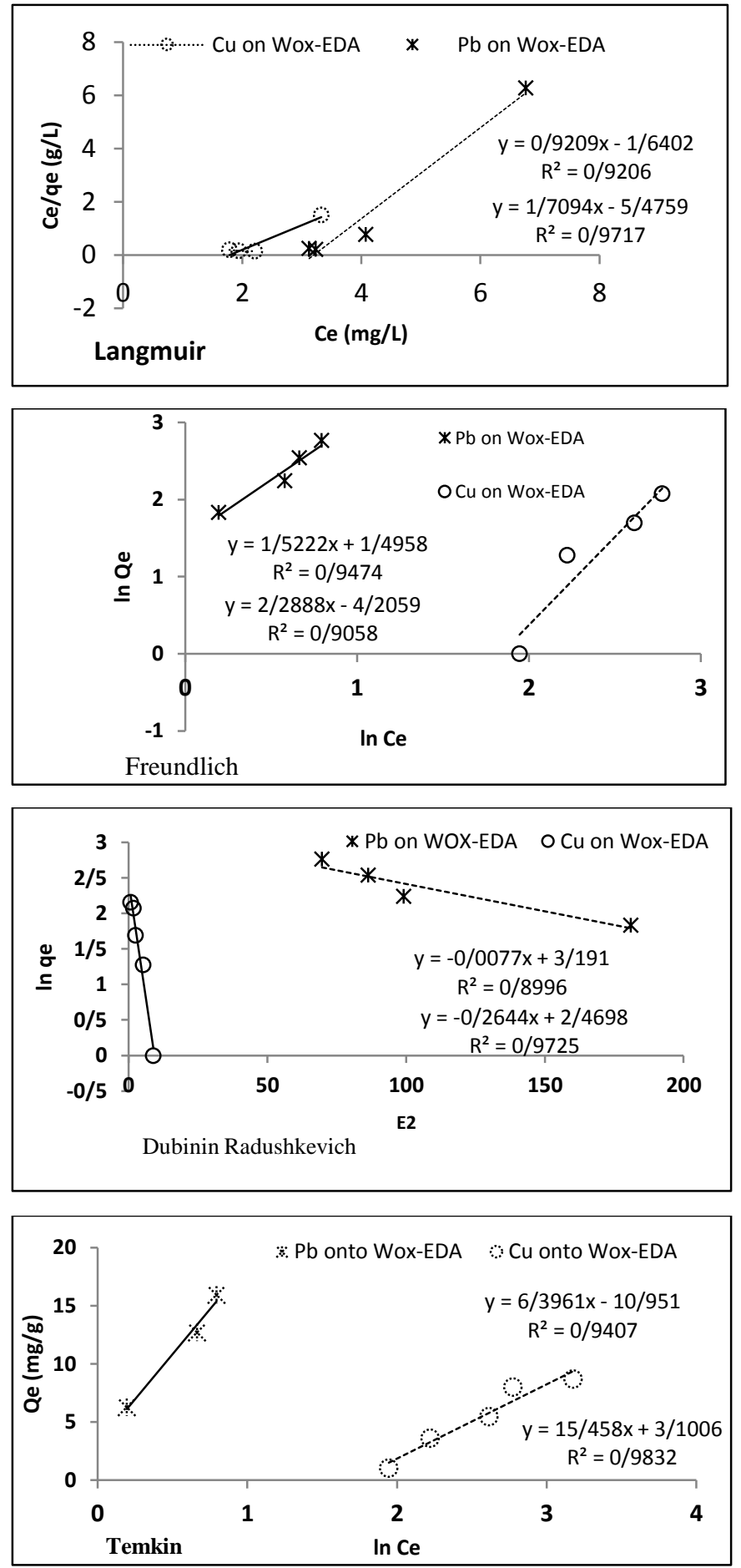

Fig. 8. Adsorption Isotherm Plots of $\mathrm{Pb}$ and $\mathrm{Cu}$ on WOx-EDA 
Table 2. Isotherm parameters showing the adsorption of $\mathrm{Pb}$ and $\mathrm{Cu}$ onto

\begin{tabular}{|l|l|l|l|}
\hline $\begin{array}{l}\text { Isotherm } \\
\text { Model }\end{array}$ & Constants & Pb-WOx-EDA & Cu-WOx-EDA \\
\hline Langmuir & $\mathrm{R}^{2}$ & 0.921 & 0.973 \\
& $\mathrm{Ka}(\mathrm{L} / \mathrm{mg})$ & 0.56 & 0.311 \\
& $\mathrm{R}_{\mathrm{L}}$ & -0.037 & -14.55 \\
Dubinin-Radushkevich & $\mathrm{Q}_{\mathrm{o}}(\mathrm{mg} / \mathrm{g})$ & 1.086 & 0.587 \\
& $\mathrm{R}^{2}$ & 0.972 & 0.899 \\
& $\left.\mathrm{~B}\left(\mathrm{~mol}^{2} / \mathrm{kJ}\right)^{2}\right)$ & 0.264 & 0.008 \\
Freundlich & $\mathrm{E}\left(\mathrm{kJ} / \mathrm{mol}^{2}\right.$ & 1.389 & 8.06 \\
& $\mathrm{R}^{2}$ & 0.947 & 0.906 \\
& $\mathrm{n}$ & 1.254 & 2.090 \\
Temkin & $\mathrm{K}_{f}(\mathrm{mg} / \mathrm{g})$ & 3.971 & 0.853 \\
& $\mathrm{R}^{2}$ & 0.983 & 0.941 \\
& $\mathrm{~B}_{\mathrm{T}}(\mathrm{KJ} / \mathrm{mol})$ & 15.45 & 10.95 \\
\hline
\end{tabular}

The Freundlich isotherm is derived by assuming a heterogeneous surface with a non-uniform distribution of heat of adsorption over the surface. The $\mathrm{R} 2$ values recorded are 0.947 and 0.906 respectively, indicating a better applicability of this model to $\mathrm{Pb}$ on WOx-EDA than $\mathrm{Cu}$ on WOx-EDA. Values recorded for ' $n$ ' and KF which represents Freundlich coefficients, are greater than 1 (Table 2). ' $n$ ' is a dimensionless factor, implying a favourable adsorption [25].

The Dubinin Radushkevich (D-R) adsorption isotherm equation is more general than the Langmuir isotherm because it does not assume a homogenous surface or constant adsorption potential. It is applied to distinguish between physical and chemical adsorption [26]. It is known that the magnitude of apparent adsorption energy, E is useful for estimating the type of adsorption. If the value of $\mathrm{E}$ is below $8 \mathrm{KJ} / \mathrm{mol}$ the adsorption type can be explained as physical adsorption, between 8 and $16 \mathrm{KJ} / \mathrm{mol}$ the adsorption type can be explained by ion exchange, and over $16 \mathrm{~kJ} / \mathrm{mol}$ the adsorption type can be explained by a stronger chemical adsorption than ion exchange. From Table 2, it is seen that $\mathrm{E}$ values for $\mathrm{Pb}$ and $\mathrm{Cu}$ onto WOx-EDA are $1.389 \mathrm{~kJ} / \mathrm{mol}$ and $8.608 \mathrm{~kJ} / \mathrm{mol}$ respectively, showing that the Adsorption type falls within the boundary of physical adsorption and ion exchange respectively. Similar results have been presented [16]

\section{Conclusion}

Versatile inorganic-organic hybrid WOx-EDA nanowires have been synthesized via a facile, low-cost, one-pot and scalable solvothermal method. This hybrid structure integrates the functionality of ethylenediamine with the stability of the WOx framework. Due to the abundance of amino groups, these WOx-EDA nanowires exhibited high affinity for a variety of heavy metal ions and displayed high adsorption capacities for $\mathrm{Pb}^{2+}$ and for $\mathrm{Cu}^{2+}$, suggesting WOx-EDA nanowires as promising drug candidate in the treatment of metal poisoning.

\section{References}

[1]G. Ivan, Medicine San frontiers, 2012, 3:1.

[2]H. Quan and X. Haiyan, Journal of Chemical and Pharmaceutical Research, 2014, 6:270.

[3]J.S.F. Swaran and P. Vidhu, International Journal of Environmental Research and Public Health, 2010, 7:2745.

[4]D. Karadag, K. Yunus, T. Mustapha and O. Mustapha, Journal of Hazardous Materials, 2007, 144:432.

[5]K. Vijayaraghavan, T. Padmesh, K. Palanivelu and M. Velan, Journal Hazardous Materials, 2006, B133:304.

[6]Y. Ho and J. Porter, Chemical Engineering Science, 2002, 24:1097.

[7]I. Igwe and A. Abia, African Journal of Biotechnology, 2006, 5:1167.

[8]Y. Kundu, Chemical Engineering Journal, 2006, 33:199.

[9]C.Y. Cao, Z.M. Cui, C.Q. Chen, W.G. Song, W. Cai, and W. Ceria, Journal of Physical Chemistry, 2010, 114:9865.

[10]M.J. Manos and M. G. Kanatzidis, Journal American.Chemical Society, 2010, 134:16441.

[11]J.A. Polleux, N. Gurlo, M.A. Weimar and M.V. Niederberger, Chemistry International Education, 2010, 45:261.

[12]C.J. Klinke, L. Hannon, K. Gignac, P. Reuter, Journal of Physical Chemistry, 2005, 109:17787.

[13]L. Wei, X. Fang, Q. Jin, L. Ping, C. Dehong, C. Zhe, Y. Yu, L. Yu, A.R. Carus and W. Song, Chemical Engineering Journal, 2014, 192:1. 
[14]J. Song, W. Zou, Y. Bian, F. Su, and R. Han Desalin, 2011, 265:119.

[15]H.Y. Naeema and A. Arkandi, International Journal of Current Microbiology and Applied Science, 2014, 4:415.

[16]K.M. Maeda, D. Higashi, R. Lu, R. Abe, and K. Domen, Journal America. Chemical Society, 2010, 132:5858.

[17]H. Xianluo, J. Qingming, P. Jonathan, and A. Katsuhiko, Desalination, 2011, 100:33.

[18]0.A. Oyedeji and G.B. sinfade, African Journal of Environmental Science and Technology, 2010, 4, 382-387.

[19]G.A. Kovo A.D. Folasegun and O.A. Kayode, Alexandria Engineering Journal, 2015, 54:757.

[20]N. Boldizsar, M. Carmen, M. Andrada, C. Indolean, Barbu-Tudoran and M. Cornelia, Arabian Journal of Chemistry, 2014, 32:554.

[21]L.Sing, A. Pavinkiemar, R. Lakshmanan and K. Rajaroo, Ecological Engineering, 2012, 1:38.

[22]G. Limousin, J. Limousin, P. Gaudet, L. Charlet, S. Szenknect, V. Barthes, M. Krimissa, Journal of Applied Geochemistry, 2007, 22:249.

[23]S.J. Allen, G. Mckay and J.F. Porter, Journal of Colloid Interface Science, 2004, 280:322.

[24]B.H. Hameed and K.Y. Foo, Chemical Engineering Journal, 2010, 156:2.

[25]P.M. Ejikeme, A.I. Okoye and O.D. Onukwuli, The African review of Physics, 2011, 6:143.

[26]A.M. Horsfall and J. Spiff, Acta Chimica Slovenica, 2005, 52: 174 . 